\title{
Quantitative Coronary Flow Capacity for Risk Stratification and Clinical Decision Making: Is It Ready for Prime Time?
}

\author{
Marcelo F. Di Carli ${ }^{1-3}$ and Rory Hachamovitch ${ }^{4}$ \\ ${ }^{I}$ Cardiovascular Imaging Program, Departments of Medicine and Radiology, Brigham and Women's Hospital, Harvard Medical \\ School, Boston, Massachusetts; ${ }^{2}$ Division of Nuclear Medicine and Molecular Imaging, Department of Radiology, Brigham and \\ Women's Hospital, Harvard Medical School, Boston, Massachusetts; ${ }^{3}$ Division of Cardiovascular Medicine, Department of Medicine, \\ Brigham and Women's Hospital, Harvard Medical School, Boston, Massachusetts; and ${ }^{4}$ Cardiovascular Imaging Section, Department \\ of Cardiovascular Medicine, Heart and Vascular Institute, Cleveland Clinic, Cleveland, Ohio
}

See the associated article on page 410.

O has been standard practice in nuclear cardiology for more than 3 decades. This approach has proven to be accurate and reproducible. Most importantly, semiquantitative measures of total perfusion deficit and myocardial ischemia are powerful markers of clinical risk and have served as clinically relevant guides to patient management. However, the notion of ischemia-guided approach to management of stable coronary artery disease (CAD) is now being revisited, and there is considerable debate regarding its role in patient management. Older observational data $(1)$ determined that a SPECTdefined threshold of approximately 10\%-15\% ischemic myocardium was associated with equipoise between early revascularization and medical therapy among a large cohort of patients without known CAD, with increasing amounts of ischemia associated with enhanced survival after revascularization. These results were extended to other populations in subsequent studies (2). On the other hand, substudies from large randomized clinical trials (3-5) comparing revascularization versus contemporary guideline-directed medical therapy (GDMT) for management of patients with stable CAD have reported conflicting results. In fact, the question of whether the presence of moderatesevere ischemia ( $>10 \%$ of the left ventricular myocardium) associates with a prognostic benefit from early referral to revascularization compared with GDMT is the subject of the ISCHEMIA trial (6), which recently completed enrollment.

Over the last $10 \mathrm{y}$, we have seen a growing interest in using quantitative myocardial blood flow (MBF) and myocardial flow reserve (MFR) as assessed by PET to improve diagnosis and management of CAD. It has been argued that such quantitative flow measurements provide a more accurate assessment of myocardial perfusion, thereby overcoming several important limitations of conventional semiquantitative perfusion imaging including

Received Dec. 16, 2018; revision accepted Dec. 19, 2018.

For correspondence or reprints contact: Marcelo F. Di Carli, Brigham and Women's Hospital, 75 Francis St., ASB-L1 037-C, Boston, MA 02115.

E-mail: mdicarli@bwh.harvard.edu

Published online Feb. 7, 2019.

COPYRIGHT (c) 2019 by the Society of Nuclear Medicine and Molecular Imaging. DOI: 10.2967/jnumed.118.219717 the underestimation of the extent of ischemia and obstructive atherosclerosis in the setting of multivessel CAD and the inability to identify patients with clinically important nonobstructive atherosclerosis. Indeed, a growing body of data have demonstrated that quantitative MBF and MFR are unique phenotyping tools to assess vascular health and preclinical atherosclerosis, which, in higher risk patients, can reveal flow-limiting coronary artery stenoses, thereby improving the accuracy of myocardial perfusion imaging in the diagnostic evaluation of known or suspected CAD (7-10). More recent data support the notion that coronary vascular dysfunction, as quantified by reduced MFR, is highly prevalent among patients with known or suspected CAD $(11,12)$, increases the severity of inducible myocardial ischemia (beyond the effects of upstream coronary obstruction) (13) and subclinical myocardial injury (14), and identifies patients at high risk for serious cardiac adverse events, including cardiac death (15-19) and heart failure (20). There is also emerging evidence that a reduced MFR may help identify patients who benefit most from revascularization (21).

In this context, the study by Gould et al. in the current issue of The Journal of Nuclear Medicine (22) sought to assess whether the measurement of the so-called coronary flow capacity (CFC), which integrates quantitative measurements of stress MBF and MFR, may provide incremental prognostic information that may potentially allow direction of care by selecting patients who would benefit most from revascularization. The study reports 3,774 patients with known or suspected CAD referred to their center for rest and stress myocardial perfusion PET imaging for the evaluation of symptoms or to assess the physiologic significance of known coronary artery stenoses. Overall, $74 \%$ of patients were nonobese men with normal left ventricular function, $41 \%$ had known CAD, and only $11 \%$ had anginal symptoms. Patients were followed for up to $9 \mathrm{y}$, with an average of $3 \mathrm{y}$ for the occurrence of all-cause death, myocardial infarction (MI), stroke, and revascularization excluding patients followed for fewer than $90 \mathrm{~d}$. The analysis was performed on a per-scan and per-patient basis with time-dependent covariates for outcomes after each PET at different times in the same patient or for different patients, indicating that the analysis included more than one PET scan and possibly more than one event per patient.

\section{DOES CFC SEVERITY PREDICT RISK?}

In risk-adjusted analyses, they found that a severe reduction in CFC (i.e., $\mathrm{CFR} \leq 1.27$ and stress $\mathrm{MBF} \leq 0.83 \mathrm{cc} / \mathrm{min} / \mathrm{g}$ ) involving $1 \%$ or more of the left ventricle was associated with a higher 
risk of major adverse cardiovascular events (MACE) including allcause death. This is not surprising given all prior data showing a prognostic association with both components of the CFC measurement (15-19). Importantly, a severe CFC was not associated with MACE after adjustment for revascularization as a time-dependent covariate (Supplemental Table 1 in Gould et al. (22)). Consistent with the extensive prior literature cited above (15-19), both regional and global reduction in MFR were also associated with the risk of MACE (Supplemental Tables 2 and 3 in Gould et al. (22)).

\section{DOES CFC SEVERITY PREDICT PROGNOSTIC BENEFIT FROM REVASCULARIZATION?}

A major goal of the study was to assess whether a severe reduction in CFC by PET could help identify patients who may benefit prognostically from revascularization. Because of the observational nature of the study design, the authors developed a propensity score to account for differences between patients undergoing revascularization and those treated medically. The propensity score included several clinical, historical, and some imaging variables including left ventricular ejection fraction and resting perfusion defect size. Surprisingly, it did not include the extent and severity of stressinduced defects or ischemia-arguably the most important determinants of referral to catheterization and revascularization along with the severity of patient symptoms (1). Their analysis showed a significant interaction between the extent of severe CFC reduction and revascularization within $90 \mathrm{~d}$ of PET imaging and reduced risk of all-cause death during follow-up. No significant interaction was observed between the extent of mild-moderate reduction in CFC or other perfusion metrics including the extent and severity of regional perfusion defects, minimal regional stress MBF or MFR, and revascularization.

On the basis of these data, the authors conclude that estimates of CFC integrating regional MFR and stress perfusion in $\mathrm{cc} / \mathrm{min} / \mathrm{g}$ by PET provide an imaging marker of clinical risk that can be used to guide revascularization decisions, and they claim that such an approach can help reduce the risk of death and myocardial infarction by $54 \%$. The strengths of the study include the relatively large number of patients with detailed quantitative PET myocardial perfusion data, the experience of the investigators in quantitative analysis of PET data, and the lengthy and robust follow-up of their cohort.

Compared with randomized clinical trials, observational studies have several advantages, including the ability of following larger, unrestricted cohorts with robust data collection for long periods of time. However, such study design requires robust statistical methods to overcome both potential confounding and the lack of treatment randomization (23). Observational studies such as this one are difficult to interpret when confounding and bias are unaccounted or partially accounted for, more the case when complex analyses are attempted (23). Thus, the current study's internal validity is undermined by numerous methodologic issues related to both multivariable model derivation and interpretation. First, 7 survival models are presented, 2 modeling all-cause death and the remainder modeling the composite of death/myocardial infarction/ stroke. In these models, severe CFC is often-but not always-a significant predictor of outcome and in one model (Table 2 in Gould et al. (22)) appears to be protective from events.

Second, the authors appropriately develop a propensity score but do not include key PET information available to referring physicians and a critical determinant of revascularization (i.e., extent of ischemia), calling into question the adequacy of this score. In fact, the revascularization group had very large stress perfusion defects involving nearly a third of the left ventricular mass $(28 \%)$ with little or no rest defects (indicating a large ischemic burden) compared with those who were treated medically (5\%). In addition, the authors included the propensity score in only one model (Table 4 in Gould et al. (22)) despite including revascularization use in all 7 models. Given the thresholds used to define a severe reduction in CFC outlined above, it would be difficult to imagine that those critical flow abnormalities would not be associated with a severe perfusion deficit, which not only is straightforward to see and measure, but it may also undermine the incremental value of the quantitative flow capacity information and the claim of such a large prognostic benefit from revascularization, which has not been realized in any of the large contemporary randomized clinical trials in stable CAD. There are other methodologic limitations including model overfit based on the relatively limited number of deaths and limited statistical power (Table 4 in Gould et al. (22)), lack of accounting for medical therapy that impacts all components of the MACE endpoint, inclusion of multiple and highly interrelated PET measurements of perfusion in their models without evidence that collinearity was examined, and repeated testing without adequate statistical adjustment. In fact, from the $P$ values in the manuscript, adjusting their results for this repeated testing would eliminate the significance of the study's primary findings.

Third, correct model interpretation is important as well. To determine whether imaging results (CFC severity) associate with a treatment benefit from an intervention (revascularization), a significant interaction between the imaging metric of interest and the intervention use must be present as part of the model. In the absence of this interaction, the statistical significance of revascularization use (with a hazard ratio $<1$ ) indicates that risk is reduced by revascularization irrespective of the values of all other variables. Hence, all but 1 model indicate that PET data were unnecessary to identify treatment benefit with revascularization. Hence the claim that "...the threshold of any CFCsevere (blue) remains as the statistically significant association with reduced death, MI or stroke after revascularization within 90 days after PET by multiple Cox regression modeling" (Supplemental Fig. 2 in Gould et al. (22)) seems unsupported. The models demonstrating statistical significance for both PET data and revascularization use do not indicate that the PET data associate with a treatment benefit after revascularization.

\section{QUANTITATIVE THRESHOLDS OF ISCHEMIA FOR GUIDING REVASCULARIZATION}

As discussed above, prior data have suggested a threshold of approximately $10 \%-15 \%$ ischemic myocardium as a point of equipoise regarding the relative benefit of revascularization versus GDMT in patients with stable CAD (24). This has informed the design of the ISCHEMIA trial, which is testing whether revascularization of patients exceeding this ischemic threshold offers a measurable outcome benefit compared with GDMT alone. The finding of insignificant hazard ratios at every level of severe CFC extent (Supplemental Fig. 2 in Gould et al. (22)) further complicates the interpretation of the study results as does the inability to identify a threshold of severe CFC extent associated with a survival benefit. This threshold is critical to inform prospective clinical trial design to test the effectiveness of this approach and, ultimately, translate the current findings into practice. Also, the current thresholds of stress MBF and MFR used in this study to define a severe CFC appear to be extremely low, which is in part related to the fact that they were derived from the development of 
clinical ischemia (ST segment depression and chest pain) during vasodilator-stress testing that is typically seen in patients with coronary steal in the setting of critical stenosis. In fact, the authors used the presence of a new stress defect as part of their definition of lowflow ischemia in the CFC measurement $(25,26)$. This suggests that if the CFC thresholds for revascularization benefit are that severely low, the incremental contribution of quantitative flow data may be more modest than described in Gould et al. (22). Other studies have reported higher thresholds $(21,27)$, which suggests that more research is necessary to define this very important point.

\section{CORONARY FLOW RESERVE, STRESS MBF, OR INTEGRATED CFC}

Stress MBF and MFR measures are clearly interrelated and highly correlated and agree with each other (concordant abnormal and concordant normal) in a large number of patients (16). Calculated as a ratio, MFR is sensitive to unusually high or low resting flow values. These 2 clinical phenotypes (unusually low and high resting flows) are precisely the groups with the largest discrepancies in risk stratification between stress MBF and MFR (16). In such scenarios, the concept of CFC, coined by the authors (26), is useful to understand pathophysiology and, more importantly, associated clinical risk. Patients with low stress flow but relatively preserved MFR are predominantly men and have a low risk of cardiac death $(<1 \% / y)$, whereas those with preserved stress flow but reduced MFR are predominantly women with a substantially higher risk of cardiac death (1\%-3\%/y) (16). The differentiation of these 2 prognostically different groups is difficult as they were lumped as mild-moderate CFC reduction in the current study. The lower risk attribution of such patients in the study is likely related to the fact that they studied a predominantly male population. It is unclear that the risk thresholds and findings described in the study are also applicable to female patients.

In summary, despite its significant limitations the study provides provocative data that should motivate further research in this area as outlined above. Ultimately, the clinical value and role of quantitative $\mathrm{CFC}$ or any other quantitative flow measurement to guide management of stable $\mathrm{CAD}$ will have to be tested in properly designed randomized clinical trials, which remain the best level of evidence we have to support clinical practice.

\section{DISCLOSURE}

No potential conflict of interest relevant to this article was reported.

\section{REFERENCES}

1. Hachamovitch R, Hayes SW, Friedman JD, Cohen I, Berman DS. Comparison of the short-term survival benefit associated with revascularization compared with medical therapy in patients with no prior coronary artery disease undergoing stress myocardial perfusion single photon emission computed tomography. Circulation. 2003;107:2900-2907.

2. Hachamovitch R, Rozanski A, Shaw LJ, et al. Impact of ischaemia and scar on the therapeutic benefit derived from myocardial revascularization vs. medical therapy among patients undergoing stress-rest myocardial perfusion scintigraphy. Eur Heart J. 2011;32:1012-1024.

3. Shaw LJ, Berman DS, Maron DJ, et al. Optimal medical therapy with or without percutaneous coronary intervention to reduce ischemic burden: results from the Clinical Outcomes Utilizing Revascularization and Aggressive Drug Evaluation (COURAGE) trial nuclear substudy. Circulation. 2008;117:1283-1291.

4. Shaw LJ, Cerqueira MD, Brooks MM, et al. Impact of left ventricular function and the extent of ischemia and scar by stress myocardial perfusion imaging on prognosis and therapeutic risk reduction in diabetic patients with coronary artery disease: results from the Bypass Angioplasty Revascularization Investigation 2 Diabetes (BARI 2D) trial. J Nucl Cardiol. 2012;19:658-669.

5. Shaw LJ, Weintraub WS, Maron DJ, et al. Baseline stress myocardial perfusion imaging results and outcomes in patients with stable ischemic heart disease randomized to optimal medical therapy with or without percutaneous coronary intervention. Am Heart J. 2012;164:243-250.

6. Bangalore S, Maron DJ, Fleg JL, et al. International Study of Comparative Health Effectiveness with Medical and Invasive Approaches-Chronic Kidney Disease (ISCHEMIA-CKD): rationale and design. Am Heart J. 2018;205:42-52.

7. Danad I, Raijmakers PG, Driessen RS, et al. Comparison of coronary CT angiography, SPECT, PET, and hybrid imaging for diagnosis of ischemic heart disease determined by fractional flow reserve. JAMA Cardiol. 2017;2:1100-1107.

8. Danad I, Uusitalo V, Kero T, et al. Quantitative assessment of myocardial perfusion in the detection of significant coronary artery disease: cutoff values and diagnostic accuracy of quantitative $\left[{ }^{15} \mathrm{O}\right] \mathrm{H} 2 \mathrm{O}$ PET imaging. $J$ Am Coll Cardiol. 2014;64:1464-1475.

9. Naya M, Murthy VL, Taqueti VR, et al. Preserved coronary flow reserve effectively excludes high-risk coronary artery disease on angiography. J Nucl Med. 2014;55: 248-255.

10. Ziadi MC, Dekemp RA, Williams K, et al. Does quantification of myocardial flow reserve using rubidium- 82 positron emission tomography facilitate detection of multivessel coronary artery disease? J Nucl Cardiol. 2012;19:670-680.

11. Murthy VL, Naya M, Foster CR, et al. Improved cardiac risk assessment with noninvasive measures of coronary flow reserve. Circulation. 2011;124:2215-2224.

12. Murthy VL, Naya M, Taqueti VR, et al. Effects of gender on coronary microvascular dysfunction and cardiac outcomes. Circulation. 2014;129:2518-2527.

13. Gould KL, Ornish D, Scherwitz L, et al. Changes in myocardial perfusion abnormalities by positron emission tomography after long-term, intense risk factor modification. JAMA. 1995;274:894-901.

14. Taqueti VR, Everett BM, Murthy VL, et al. Interaction of impaired coronary flow reserve and cardiomyocyte injury on adverse cardiovascular outcomes in patients without overt coronary artery disease. Circulation. 2015;131:528-535.

15. Fukushima K, Javadi MS, Higuchi T, et al. Prediction of short-term cardiovascular events using quantification of global myocardial flow reserve in patients referred for clinical ${ }^{82} \mathrm{Rb}$ PET perfusion imaging. J Nucl Med. 2011;52:726-732.

16. Gupta A, Taqueti VR, van de Hoef TP, et al. Integrated noninvasive physiological assessment of coronary circulatory function and impact on cardiovascular mortality in patients with stable coronary artery disease. Circulation. 2017;136:2325-2336.

17. Herzog BA, Husmann L, Valenta I, et al. Long-term prognostic value of ${ }^{13} \mathrm{~N}$ ammonia myocardial perfusion positron emission tomography added value of coronary flow reserve. J Am Coll Cardiol. 2009;54:150-156.

18. Murthy VL, Naya M, Foster CR, et al. Non-Invasive Quantitative Assessment of Coronary Flow Reserve Improves Cardiac Risk Assessment in Patients with Diabetes. Circulation. 2011;124:2215-2224.

19. Ziadi MC, Dekemp RA, Williams KA, et al. Impaired myocardial flow reserve on rubidium- 82 positron emission tomography imaging predicts adverse outcomes in patients assessed for myocardial ischemia. J Am Coll Cardiol. 2011;58:740-748.

20. Taqueti VR, Solomon SD, Shah AM, et al. Coronary microvascular dysfunction and future risk of heart failure with preserved ejection fraction. Eur Heart J. 2018;39:840-849.

21. Taqueti VR, Hachamovitch R, Murthy VL, et al. Global coronary flow reserve is associated with adverse cardiovascular events independently of luminal angiographic severity and modifies the effect of early revascularization. Circulation. 2015;131:19-27.

22. Gould KL, Johnson NP, Roby AE, et al. Regional, artery-specific thresholds of quantitative myocardial perfusion by PET associated with reduced myocardial infarction and death after revascularization in stable coronary artery disease. J Nucl Med. 2019;60:410-417.

23. Hachamovitch R, Di Carli MF. Methods and limitations of assessing new noninvasive tests: part II-outcomes-based validation and reliability assessment of noninvasive testing. Circulation. 2008;117:2793-2801.

24. Gibbons RJ, Miller TD. Should extensive myocardial ischaemia prompt revascularization to improve outcomes in chronic coronary artery disease? Eur Heart J. 2015;36:2281-2287.

25. Johnson NP, Gould KL. Physiological basis for angina and ST-segment change PET-verified thresholds of quantitative stress myocardial perfusion and coronary flow reserve. JACC Cardiovasc Imaging. 2011;4:990-998.

26. Johnson NP, Gould KL. Integrating noninvasive absolute flow, coronary flow reserve, and ischemic thresholds into a comprehensive map of physiological severity. JACC Cardiovasc Imaging. 2012;5:430-440.

27. Driessen RS, Danad I, Stuijfzand WJ, et al. Impact of revascularization on absolute myocardial blood flow as assessed by serial $\left[{ }^{15} \mathrm{O}\right] \mathrm{H} 2 \mathrm{O}$ positron emission tomography imaging: a comparison with fractional flow reserve. Circ Cardiovasc Imaging. 2018;11:e007417. 